\title{
Favorable renal outcome in Japanese children with ANCA-associated pauci-immune glomerulonephritis: authors' response to comments
}

\author{
Anne M. Kouri ${ }^{1}$ • Sharon P. Andreoli ${ }^{1}$ \\ Received: 28 October 2016 / Accepted: 2 November 2016 / Published online: 24 November 2016 \\ (C) IPNA 2016
}

\section{Dear Sir,}

We would like to thank Drs Fujinaga and Nishino [1] for their thoughtful response to our retrospective study of children with ANCA-associated glomerulonephritis at our single center.

First, we would like to compliment them on their favorable long-term outcomes in their patients. We also agree that earlier detection of urinary abnormalities by routine screening of all school-age children does allow for earlier detection of this rare disease. This is supported by a trend toward an earlier median age of presentation of 7.3 years in the aforementioned Japanese cohort as compared to 13.7 years in our study [2].

Additionally, in a study of 31 Japanese patients by Hattori et al. published in 2001, nine patients (29\%) developed endstage renal disease (ESRD) [3]. These patients with ESRD tended to have a longer latency period between disease onset and diagnosis, and they were less likely to have their disease detected by urine screening. However, these differences were not statistically significant between those patients with ESRD and those patients with normal renal function [3]. As such, early detection likely plays a role in improved renal outcomes despite similar immunosuppression treatment protocols between the U.S. and Japanese cohorts. However, we also recognize that there are potentially other factors that could be further explored that may contribute to the outcome discrepancies, including myeloperoxidase versus proteinase 3 positivity, gender differences, as well as ethnic and environmental factors.

Compliance with ethical standards

Conflict of interest The authors declare that there is no conflict of interest.

\section{References}

1. Fujinaga S, Nishino T (2016) Favorable renal outcome in Japanese children with ANCA-associated pauci-immune glomerulonephritis. Pediatr Nephrol. doi:10.1007/s00467-016-3542-y

2. Kouri AM, Andreoli SP (2016) Clinical presentation and outcome of pediatric ANCA-associated glomerulonephritis. Pediatric nephrology. doi:10.1007/s00467-016-3490-6

3. Hattori M, Kurayama H, Koitabashi Y, Japanese Society for Pediatric Nephrology (2001) Antineutrophil cytoplasmic autoantibodyassociated glomerulonephritis in children. J Am Soc Nephrol 12: $1493-1500$

Anne M. Kouri

akouri@iupui.edu

1 Department of Pediatric Nephrology, Indiana University School of Medicine, 699 Riley Hospital Drive, Rm 230,

Indianapolis, IN 46202, USA 